\title{
Discrete optimal capital investment and financing policies in fishery resource harvesting with reserve area
}

\author{
Muhamad Nagib Alatas*, Toni Baktiar, Farida Hanum \\ Division of Operation Research, Department of Mathematics, Bogor Agricultural \\ University, Kampus IPB Dramaga, Bogor 16680, INDONESIA \\ *Corresponding author: s.n.alatas@gmail.com
}

\begin{abstract}
This research discusses a problem in renewable resource (fish) harvesting on single-owner fishery where the dynamics of growth are expressed by system of difference equations. The model involves three state variables (stock of equity, resource stock in reserve zone and unreserved zone) and two control variables (effort rate and dividend payout rate). Discrete Pontryagin maximum principle was applied to obtain optimality conditions which must be satisfied by the variables. Further, the forward-backward sweep method was used to determine the numerical solution of optimal control model. Three scenarios of interest rate applied to find out the response of company in managing harvesting effort and dividend payout rate were investigated.
\end{abstract}

\section{Introduction}

A fishery is all about catching, maintenance, and cultivation of fish. The characteristic of rightful ownership of fishery resource is a common property that leads to an open-access fishery. In openaccess fishery, profit tends to decrease and stocks may be extinct. To prevent overfishing that can lead to the extinction of stocks, there are many efforts have been applied including the development of mathematical approaches to make best decisions.

Clark et al. (1979) discussed optimal harvesting at the renewable resource (fish) for a fish company with irreversible investment assumption. Jørgensen and Kort (1997) discussed optimal harvesting in renewable resource, which allowed the firm to have a debt or lend with the interest rate depend on the firm's cumulative amount of debt or lending level and replaced irreversible investment assumption with perfectly reversible investment assumption. Dubey et al. (2003) introduced and analyzed model of resource (fish) with allocation zone, that is reverse zone and non-reserve zone. The harvesting allowed at the non-reserve zone. The three models above are continuous types, but in the real world, the continuous model is less realistic because the decision will be made at a discrete and probably periodic time.

In this work we present an optimal harvesting model of a renewable natural resource. We offer the discrete-time version of Jorgensen and Kort (1997) of optimal investment and finance in renewable resource harvesting and that of Dubey et al. (2003) of fishery resource dynamic with reserve area. In this model, three state variables (stock of equity, resource stock in reserve zone and non-reserve zone) and two control variables (effort rate and dividend payout rate) were introduced. It is shown that company has a debt when the capital or stock of equity is not enough for harvesting, and when the capital or stock of equity is enough for harvesting, the company will stop to debt and start to give a lend. In the meantime the company is always make dividend payment. 


\section{Optimal control problem}

Let $S(k)$ denotes the stock of fishery resource at non-reserve area by time $k, V(k)$ represents the stock of fishery resource at reserve area by time $k$ and $X(k)$ refers to the stock of equity at time $k$, where $k=1,2, \ldots, N$. The discrete dynamical model of fishery resource management at reserve area that combine the harvesting effort, capital investment and financing policies can be given as follow:

$$
\begin{aligned}
& S(k+1)-S(k)=\alpha S(k)\left(1-\frac{S(k)}{M}\right)-r_{1} S(k)+r_{2} V(k)-q E(k) S(k), \\
& V(k+1)-V(k)=\beta V(k)\left(1-\frac{V(k)}{L}\right)+r_{1} S(k)-r_{2} V(k), \\
& X(k+1)-X(k)=(p q S(k)-c-m a) E(k)-C(B(k))-D(k),
\end{aligned}
$$

In the above model, we define by $E(k)$ and $D(k)$ the harvesting effort rate and dividend payout rate by time $k$, respectively. The cost of fund $C$ is assumed to be a function of the level of debt/lending by time $k, B(k)$. Thus implicitly we have $B(k)=m E(k)-X(k)$, where $m$ is the unit cost of harvesting effort. When $X>m E(B<0)$ the firm has excess equity that leads to the firm to give a lending, and when $X<m E(B>0)$ the cost of harvesting effort more than equity that leads to the firm to debt. In model (1), $\alpha, \beta, r_{1}, r_{2}, p, q, c, M$ and $L$ are all parameters.

The control problem is to find control variables $E$ and $D$ such that maximized the present value of dividend payout over an infinite horizon $J=\int_{0}^{\infty} D(t) e^{-i t} d t$ that can further be expressed by

$$
\max J=\sum_{k=0}^{\infty}(1+i)^{-k}[(p q S(k)-c-m a) E(k)-C(B(k))-i X(k)],
$$

where $i$ is the market interest rate. In this problem it is assumed that all control variables are bounded, i.e., $E_{\min } \leq E(k) \leq E_{\max }$ and $D_{\min } \leq D(k) \leq D_{\max }$ for all time $k$. It is also assumed that $S(0)=S_{0}, V(0)=V_{0}, X(0)=X_{0}$ and $S(N), V(N), X(N)$ are all free.

We take into account two intervention strategies, namely fish harvesting and dividend payment. Three cases were considered relating to the choice of cost of fund function:

Linear

piecewise linear

$$
C(B(k))=z B(k)
$$

and quadratic

$$
C(B(k))= \begin{cases}z_{1} B(k) & ; B(k) \leq 0 \\ z_{2} B(k) & ; B(k)>0\end{cases}
$$

$$
C(B(k))= \begin{cases}z_{1} B(k) & ; B(k) \leq 0 \\ z_{1} B(k)+z_{3} B^{2}(k) & ; B(k)>0 .\end{cases}
$$

Here, $z_{1}, z_{2}$ and $z_{3}$ are positive constants related to interest rate for either debt or lend. In the linear case it is obvious that the interest rate is constant and the same for debt or lending. For the piecewise linear case the interest rate is constant but different for debt or lending. For the quadratic case, the interest rate is constant for lending and quadratic for debt that means the interest rate will increase related to the debt level.

\section{Optimality conditions}

We generate the necessary condition for the optimal control by using the discrete version of Pontryagin Maximum Principle. We need to define the Hamiltonian $H$ based on (2) subject to system state (1) to obtain the necessary conditions: 


$$
\begin{aligned}
H= & \frac{1}{(1+i)^{k}}[(p q S(k)-c-m a) E(k)-C(B(k))-i X(i)] \\
& +P_{1}(k)\left[\alpha S(k)\left(1-\frac{S(k)}{M}\right)-r_{1} S(k)+r_{2} V(k)-q E(k) S(k)\right] \\
& +P_{2}(k)\left[\beta V(k)\left(1-\frac{V(k)}{L}\right)+r_{1} S(k)-r_{2} V(k)\right] \\
& +P_{3}(k)[(p q S(k)-c-m a) E(k)-C(B(k))-D(k)],
\end{aligned}
$$

with $P_{1}(k), P_{2}(k)$ and $P_{3}(k)$ are adjoin functions to be optimally selected. The optimality conditions insisted by Pontryagin Maximum Principle are: (i) $H_{E}=0, H_{D}=0$, (ii) $P_{i}(k)-P_{i}(k-1)=$ $-H_{x_{i}}$, where $x_{i} \in\{S, V, X\}$, and (iii) $x_{i}(k+1)-x(k)=f_{i}$, where $f_{i}$ is the right hand side of system state (1), respectively.

The optimal system of equation (1) is reclaimed by applying the third condition of Pontryagin Maximum Principle, i.e., by taking the appropriate partial derivative of Hamiltonian (6) with respect to the associated state variables. Furthermore, we are able to determine the optimal control variables $E^{*}, D^{*}$ by imposing the first principle, i.e., $H_{E}=0, H_{D}=0$. The optimal controls for every cases of cost of fund functions are given as follow.

Linear:

with

$$
E^{*}= \begin{cases}E_{\max } & ; \sigma>0 \\ E_{\min } & ; \sigma<0\end{cases}
$$

Piecewise linear:

$$
\sigma=\left(\frac{1}{(1+i)^{k}}+P_{3}(k)\right)[(p q S(k)-c-m a)-z]-P_{1}(k)(q S(k)) .
$$

with

$$
E^{*}= \begin{cases}E_{1 \max } & ; B(k) \leq 0, \sigma_{1}>0 \\ E_{1 \min } & ; B(k) \leq 0, \sigma_{1}<0 \\ E_{2 \max } & ; B(k)>0, \sigma_{2}>0 \\ E_{2 \min } & ; B(k)>0, \sigma_{2}<0\end{cases}
$$

Quadratic:

$$
\sigma_{i}=\left(\frac{1}{(1+i)^{k}}+P_{3}(k)\right)\left[(p q S(k)-c-m a)-z_{i}\right]-P_{1}(k)(q S(k)) .
$$

with

$$
E^{*}= \begin{cases}E_{1 \max } & ; B(k) \leq 0, \sigma>0 \\ E_{1 \min } & ; B(k) \leq 0, \sigma<0 \\ E_{2 \min } & ; B(k)>0, E_{2}{ }^{*}<E_{2 \min } \\ E_{2}{ }^{*} & ; B(k)>0, E_{2} \min \leq E_{2}{ }^{*} \leq E_{2 \max } \\ E_{2 \max } & ; B(k)>0, E_{2}{ }^{*}>E_{2 \max }\end{cases}
$$

and

$$
\sigma=\left(\frac{1}{(1+i)^{k}}+P_{3}(k)\right)\left(p q S(k)-c-m a-z_{1}\right)-q P_{1}(k) S(k),
$$

where

$$
E_{2}^{*}=\frac{1}{m}\left(\frac{A-z_{1}}{2 z_{3}}+X(k)\right)
$$

$$
A=p q S(k)-c-m a-\frac{q P_{1}(k) S(k)}{(1+i)^{-k}+P_{3}(k)} .
$$

Since Hamiltonian is linear with respect to control function $D$, then the optimal control $D^{*}$ for every cases is given by the following bang-bang control 
with

$$
D^{*}=\left\{\begin{array}{ll}
D_{\max } & ; \tau>0 \\
D_{\min } & ; \tau<0
\end{array},\right.
$$

$$
\tau=-P_{3}(k) .
$$

The second principle of Pontryagin's Maximum is stated as follows. Given optimal controls $E^{*}, D^{*}$ and corresponding solutions to the system states $S^{*}, V^{*}, X^{*}$ that maximized objective function (2), there exists functions $P_{1}, P_{2}$ and $P_{3}$ that satisfying the system of adjoin below

$$
\begin{aligned}
& P_{1}(k-1)= P_{1}(k)\left[\alpha\left(1-\frac{2 S(k)}{M}\right)-r_{1}-q E(k)+1\right] \\
&+\left(\frac{1}{(1+i)^{k}}+P_{3}(k)\right) p q E(k)+P_{2}(k) r_{1},
\end{aligned}
$$

Since $S(N), V(N), X(N)$ are all free, the following transversality conditions must be satisfied by adjoin functions:

$$
P_{1}(N)=0, P_{2}(N)=0, P_{3}(N)=0 .
$$

\section{Results and discussion}

In this section, we discuss the numerical solution and the optimal control of the optimal system. An iterative forward method is used for solving the state system and the iterative backward method is used for solving the adjoin system. We implement this procedure based on the parameter values.

Table 1 present a value of parameter used in the numerical simulation of the model. Due to lack of data, we make a hypothesis for the parameter value within realistic range. The initial value for the state are given by $S_{0}=7500, V_{0}=9000$, and $X_{0}=10$.

We start comparing the case of the optimal harvesting with three kind of cost of fund function: linear, piecewise linear and quadratic. In Figure 1, the fish population shown in blue line and the red line is environmental carrying capacity. It is shown that under control the carrying capacity of environment in both zones are maintained. The opposite situation happened in unreserved zone when no control was applied. In Figure 2, we shown the optimal harvesting with respect to the type of cost of fund function: linear, piecewise linear and quadratic, respectively. At the end, the value of optimal harvesting is similar, that is in the maximum level of harvesting $E=19$. In Figure 3, we shown that the company always make dividend payment with the results are similar for every cases. In Figure 4, we shown the value of equity and debt/lending level, respectively. The value of equity and debt/lending level is similar for every cases, that in the beginning the company have negative equity which mean expenditure more than income, and the company have a debt, at the some point the equity will be positive and the company have a lend. Figures 5-7 depict the adjoin functions and their satisfactions on the final time conditions (8). While, Figures 8-10 illustrate the switching functions $\sigma$ and $\tau$. 
Table 1. Value of parameter

\begin{tabular}{|c|c|c|}
\hline Parameter & Value & Unit \\
\hline$\alpha$ & 0.5 & $\%$ per year \\
\hline$\beta$ & 0.6 & $\%$ per year \\
\hline$r_{1}$ & 0.2 & $\%$ per year \\
\hline$r_{2}$ & 0.25 & $\%$ per year \\
\hline$M$ & 416304 & Ton \\
\hline$L$ & 450000 & Ton \\
\hline$q$ & 0.00456 & $\%$ per ship \\
\hline$p$ & 0.1 & IDR $10^{7} /$ ton \\
\hline$c$ & 3 & IDR $10^{7} / \mathrm{ship}$ \\
\hline$a$ & 0.05 & $\%$ per year \\
\hline$m$ & 10 & IDR $10^{7} /$ ship \\
\hline$i$ & 0.08 & $\%$ per year \\
\hline$z_{1}$ & 0.03 & $\%$ per year \\
\hline$z_{2}$ & 0.11 & $\%$ per year \\
\hline$z_{3}$ & 0.0002 & $\%$ per year \\
\hline$E_{\min }($ Linear) & 2 & Ship per year \\
\hline$E_{\max }$ (Linear) & 19 & Ship per year \\
\hline$E_{1 \min }$ (Piecewise linear) & 11 & Ship per year \\
\hline$E_{1 \max }$ (Piecewise linear) & 19 & Ship per year \\
\hline$E_{2 \min }$ (Piecewise linear) & 2 & Ship per year \\
\hline$E_{2 \max }$ (Piecewise linear) & 10 & Ship per year \\
\hline$E_{1 \min }$ (Quadratic) & 2 & Ship per year \\
\hline$E_{1 \max }$ (Quadratic) & 19 & Ship per year \\
\hline$E_{2 \min }$ (Quadratic) & 2 & Ship per year \\
\hline$E_{2 \max }$ (Quadratic) & 30 & Ship per year \\
\hline$D_{\min }$ & 0 & IDR $10^{7}$ \\
\hline$D_{\max }$ & 100 & IDR $10^{7}$ \\
\hline
\end{tabular}
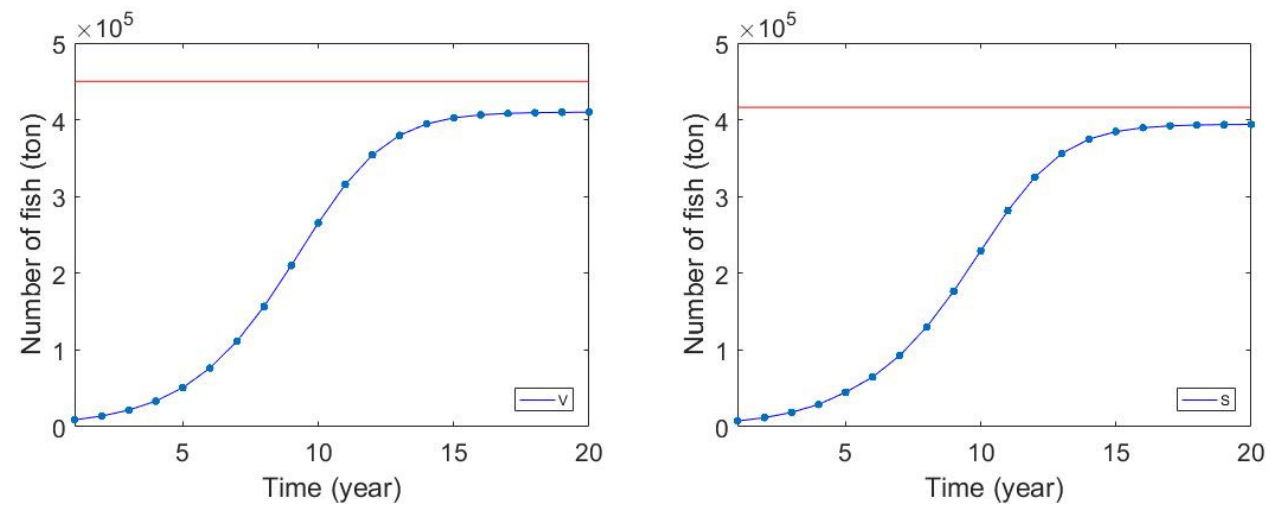

Figure 1. Fish population at reserve area (left) and non-reserve area (right) 

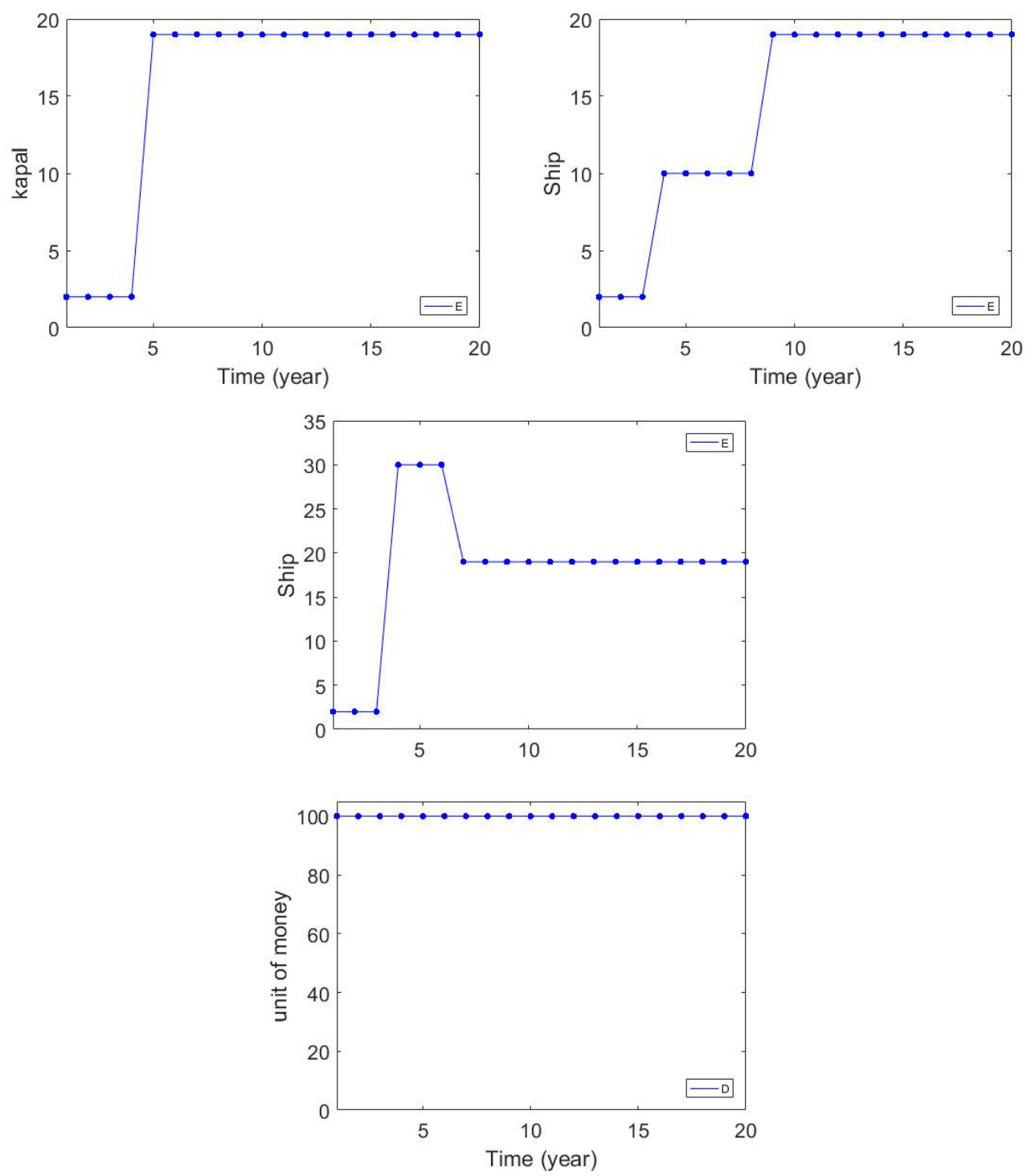

Figure 3. Optimal dividend payment
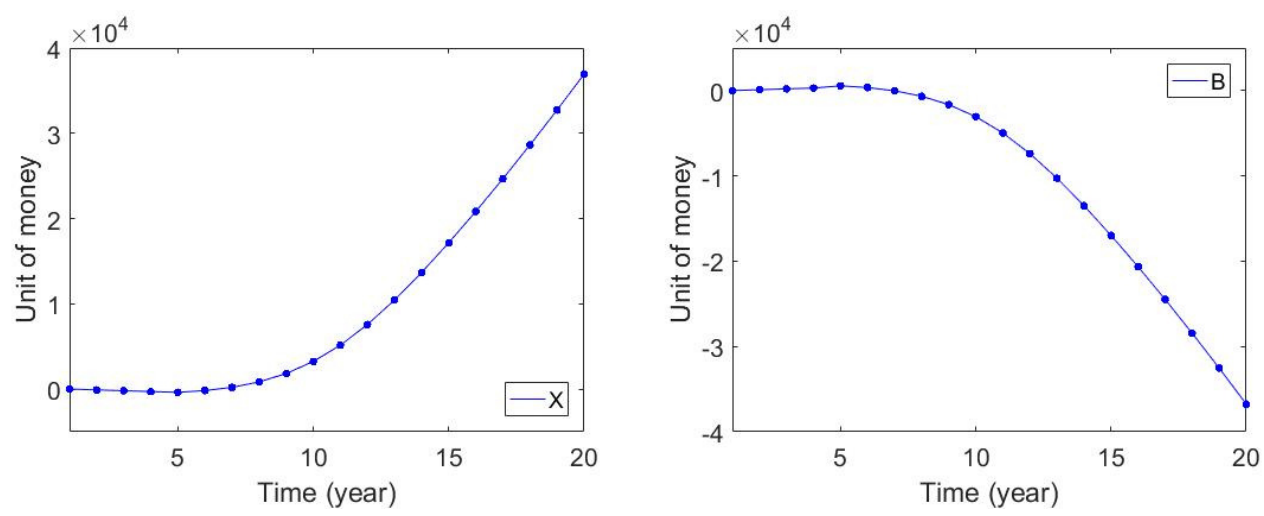

Figure 4. Equity (left) and Debt/lending level (right) 

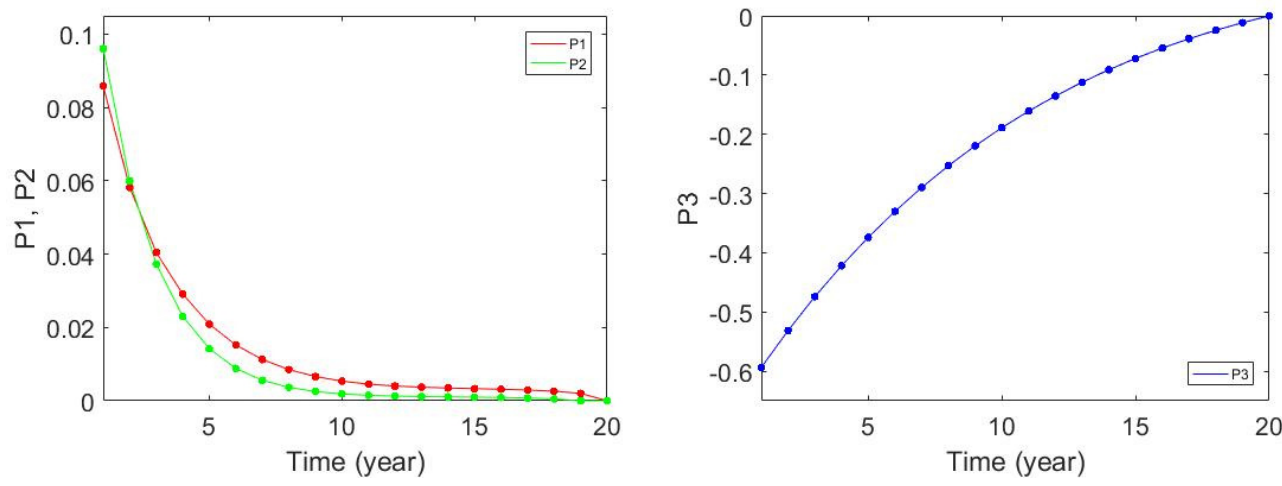

Figure 5. Adjoin function (linear case)
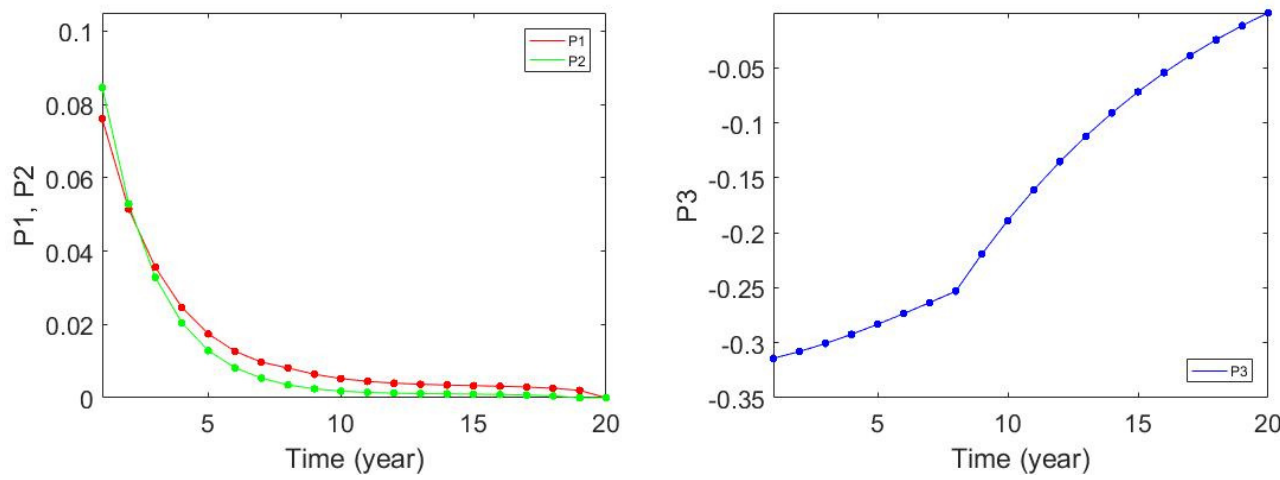

Figure 6. Adjoin function (piecewise linear case)
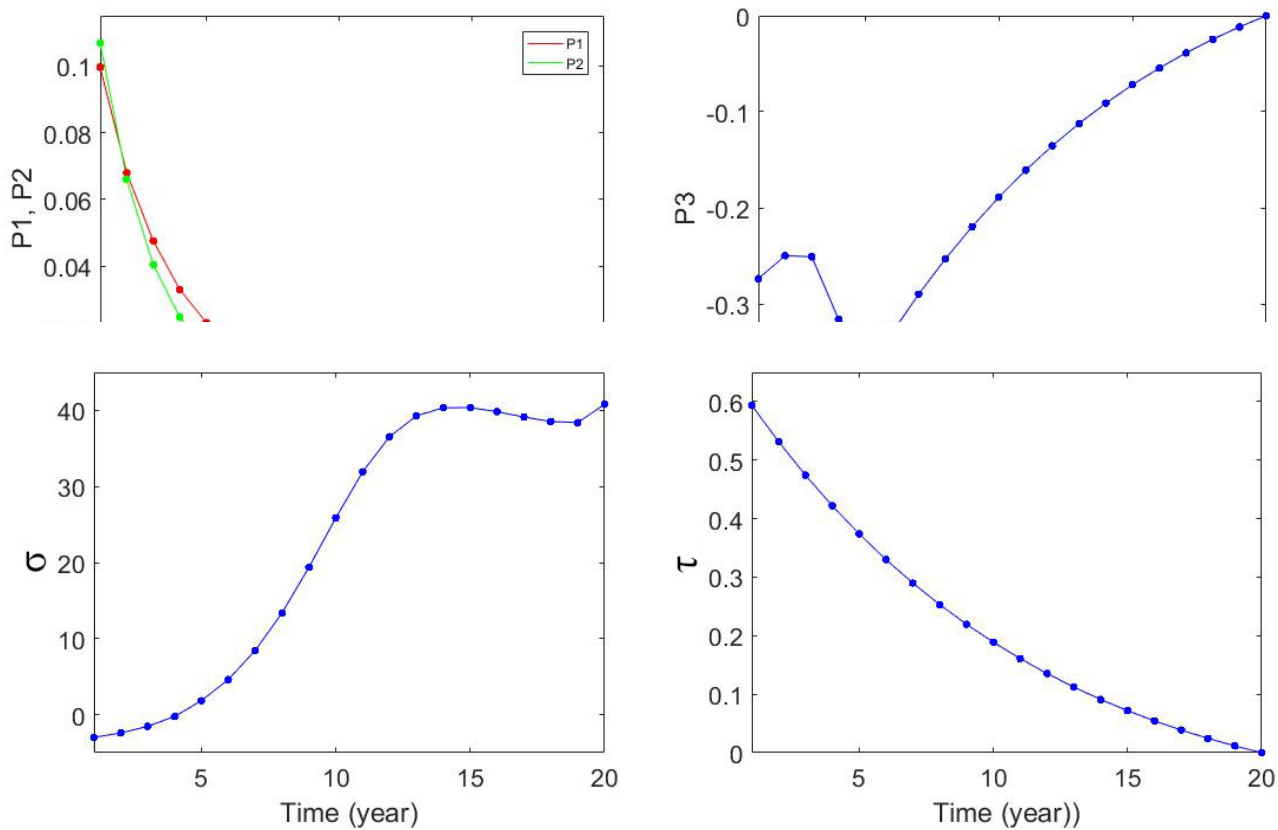

Figure 8. Harvesting and dividend payment switching function (linear case) 

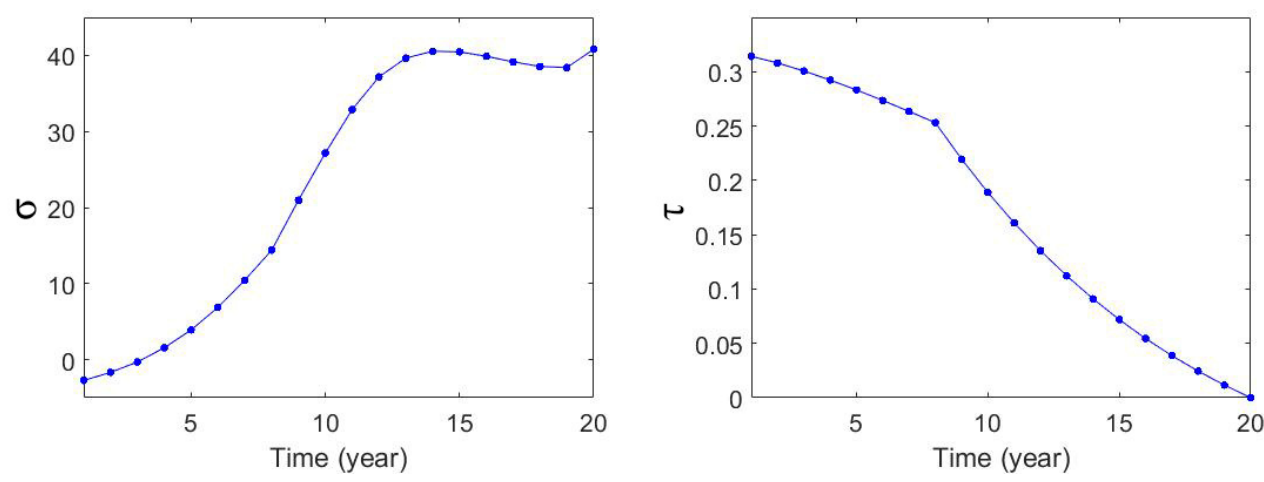

Figure 9. Harvesting and dividend payment switching function (piecewise linear case)
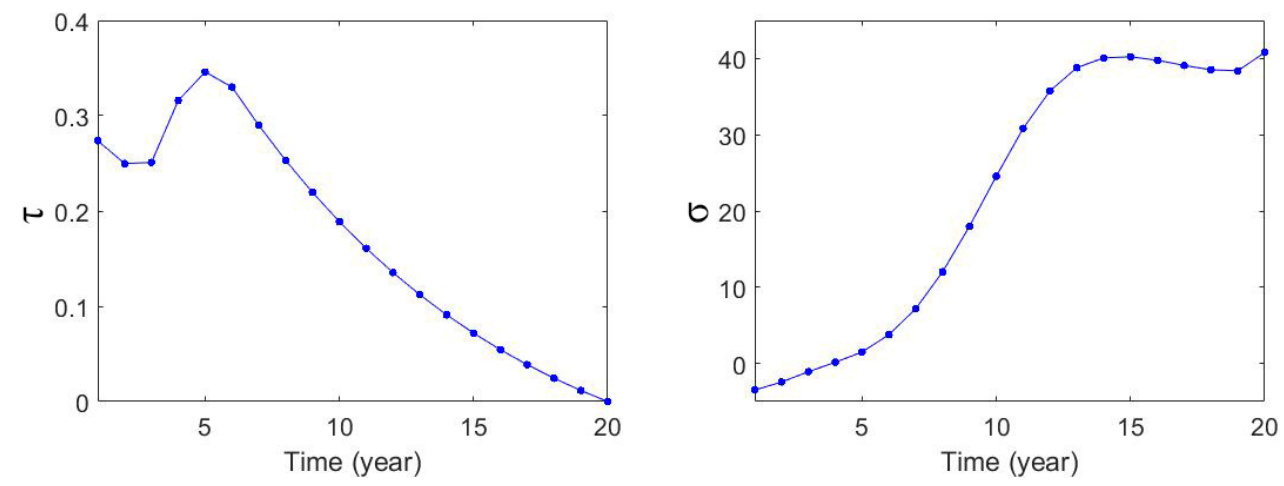

Figure 10. Harvesting and dividend payment switching function (quadratic case)

\section{Conclusion}

In this work, we have discussed a simple mathematical model of optimal harvesting in renewable resource (fish) involved capital investment and financing policies, with reserve and non-reserve area of harvesting. Based on numerical simulation, the resource without harvesting that leads to over population exceed the environmental carrying capacity so the harvesting effort is needed.

Optimal harvesting effort and dividend payout was determine by discrete version of Pontryagin maximum principle. At the time $k=1$ for every cases, the firm need to have a debt for doing harvesting and the firm has to pay the interest, at the time $k=7, k=9, k=7$ for linear, piecewise linear and quadratic case, respectively, the firm has paid off the debt and start giving a lend and receive an interest payment that leads to increase the value of equity dramatically, in the meantime the company is always make dividend payment

\section{Acknowledgments}

Authors gratefully acknowledge financial support provided by Ministry of Research, Technology and Higher Education of the Republic of Indonesia under grant No. 011/SP2H/LT/DRPM/IV/2017 through the PUPT Scheme of Bogor Agricultural University.

\section{References}

[1] Clark CW, Clarke FH, Munro GR, The optimal exploitation of renewable resource stocks: problems of irreversible investment. Econometrica, 47(1): 25-47 (1979). 
[2] B. Dubey, P. Chandra and P. Sinha, A model for fishery resource with reserve area. Nonlinear Analysis: Real World Application, 4: 625-637 (2003).

[3] S. Jørgensen and P.M. Kort, Optimal investment and finance in renewable resource harvesting. Journal of Economic Dynamics and Control, 21: 603-630 (1997).

[4] PNV Tu. Introductory Optimization Dynamics Optimal Control with Economics and Management Science Applications. New York (US): Springer (1984). 